CIENCIAMATRIA

Revista Interdisciplinaria de Humanidades, Educación, Ciencia y Tecnología

Año VI. Vol. VI. N¹. Edición Especial. 2019

Hecho el depósito de ley: pp201602FA4721

ISSN-L: 2542-3029; ISSN: 2610-802X

Universidad Nacional Experimental Francisco de Miranda (UNEFM). Santa Ana de Coro. Venezuela

Dubal Edisson Salvatierra Tumbaco; Patricio Alfredo Vallejo Valdivieso; María Elena Moya Martínez

DOI $10.35381 / \mathrm{cm} . \mathrm{v} 5 \mathrm{i} 1.260$

\title{
Estructuras mentales y competencias emocionales en estudiantes universitarios
} Mental structures and emotional competencies in university students

\author{
Dubal Edisson Salvatierra Tumbaco \\ dsalvatierra2012@gmail.com \\ Pontificia Universidad Católica del Ecuador, Extensión Manabí, Portoviejo \\ Ecuador \\ https://orcid.org/0000-0003-0042-1334 \\ Patricio Alfredo Vallejo Valdivieso \\ pvallejo@utm.edu.ec \\ pvallejo@pucem.edu.ec \\ Universidad Técnica de Manabí \\ Pontificia Universidad Católica del Ecuador, Extensión Manabí, Portoviejo \\ Ecuador \\ María Elena Moya Martínez \\ mmoya@pucem.edu.ec \\ Pontificia Universidad Católica del Ecuador, Extensión Manabí, Portoviejo \\ Ecuador \\ https://orcid.org/0000-0001-7504-6933
}

Recibido: 29 de septiembre de 2019

Aprobado: 30 de octubre de 2019

\section{RESUMEN}

La investigación tiene por objetivo determinar la relación entre estructuras mentales y competencias emocionales en estudiantes de la carrera de enfermería de la universidad Técnica de Manabí, sede Portoviejo - Ecuador. Se desarrolló mediante la correlación de Pearson, se aplicó un instrumento de 41 ítems de cinco alternativas de repuestas en escala tipo Likert a 21 estudiantes. Los resultados entre estructuras mentales (EM) y competencias emocionales (CE), demuestran una relación positiva baja de ,183. Se generan una serie de actividades que permiten al estudiante conciliar no solo teoría y significación social a los fenómenos abordados en el aprendizaje, sino, también el 
fomento de una adecuada personalidad promovida desde lo emocional como ente que permite configurar la integralidad de la persona en consonancia con ser reflexivo, critico. Descriptores: Neuropsicología, investigación sobre el cerebro, educación alternativa, experimento educacional.

\begin{abstract}
The research aims to determine the relationship between mental structures and emotional competences in students of the nursing career of the Technical University of Manabí, Portoviejo headquarters - Ecuador. It was developed through Pearson's correlation, an instrument of 41 items of five alternatives of Likert-type responses was applied to 21 students. The results between mental structures (MS) and emotional competences (CE), show a low positive relationship of, 183. A series of activities are generated that allow the student to reconcile not only theory and social significance to the phenomena addressed in learning, but also the promotion of an appropriate personality promoted from the emotional as an entity that allows to configure the integrality of the person accordingly With being thoughtful, critical.
\end{abstract}

Descriptors: Neuropsychology, brain research, alternative education, educational experiments.

\title{
INTRODUCCIÓN
}

La educación viene adoptando la posibilidad de incorporar diversos enfoques pedagógicos con la finalidad de promover el aprendizaje significativo en funcionalidad con ser pensante - actuante en el entorno social, así se habla de aprender a lo largo de la vida, involucrándose una serie de procesos donde la neurodidáctica ha sido planteada como alternativa para complementar tal funcionabilidad, correspondiéndose a la estimulación de las estructuras mentales como foco generador de un pensador mediático y crítico, al respecto Briceño Ruperti, Vallejo Valdivieso \& Moya Martínez (2019), señalan que es posible cuando se combina además el fomento de lo emocional como un factor protagónico de la articulación de una personalidad integral, correspondiéndose a la formación de un ser bio psico social espiritual.

Para lo cual se requiere de contar con una visión docente transformadora por cuanto es necesario actuar desde una perspectiva sistémica del aprendizaje que transcienda la 
visión tradicional, Morgado, Aldana Zavala \& Isea Argüelles (2019), indican la necesidad de la existencia de una gerencia educativa que contribuya al fomento del aprendizaje desde un vertiente holista, conectándose la estimulación cerebral para configurar un proceso educativo relacionado al crecimiento integral de la persona, por tal motivo, la presente investigación tiene por objetivo determinar la relación entre estructuras mentales y competencias emocionales en estudiantes de la carrera de enfermería de la universidad Técnica de Manabí, sede Portoviejo - Ecuador.

\section{DESARROLLO}

\section{Estructuras mentales y competencias emocionales}

El hablar de competencias emocionales involucra en cierto modo, conocer parte del funcionamiento cerebral de cómo se involucran las estructuras mentales racionales y emocionales en conjugación para gestar el conocimiento, así como se desarrolla la adquisición del aprendizaje progresivamente en la medida que el ser humano evoluciona biológicamente, en cuanto a las competencias emocionales, Bisquerra (2007), declara que:

Entendemos las competencias emocionales como el conjunto de conocimientos, capacidades, habilidades y actitudes necesarias para tomar conciencia, comprender, expresar y regular de forma apropiada los fenómenos emocionales. La finalidad de estas competencias se orienta a aportar valor añadido a las funciones profesionales y promover el bienestar personal y social. Las competencias emocionales son un aspecto importante de la ciudadanía activa, efectiva y responsable. (p. 1).

Así se genera las competencias en la medida que la persona va adquiriendo hábitos, los cuales podrían ser producto de entrenamientos o de aplicación de estrategias que permitan configurar acciones progresivas de consecución en el caso de estudio del aprendizaje significativo, siendo importante, estudiar la adquisición del conocimiento. Desde la visión de Bisquerra (2007), se generan cinco competencias:

1. Conciencia emocional 


\section{Regulación emocional}

3. Autonomía emocional

4. Competencia social

5. Competencias para la vida y el bienestar

Las competencias se complementan entre sí para promover la adquisición del conocimiento desde una concepción que configura la persona la posibilidad de equilibrar desde el accionar social, la interacción entre los sujetos y los significados a los fenómenos permiten articular un accionar que favorezca la adquisición del aprendizaje desde una vertiente significativa, siendo interesante conocer:

\section{¿Cuándo empieza el aprendizaje?}

En este sentido, Lagos (2014), manifiesta que: el aprendizaje empieza entes de nacer, y muchas cosas de gran importancia que aprende el ser humano, lo hace en el vientre de la madre, que las experiencias que viva el feto dentro del vientre de su madre definirán en gran cantidad la personalidad que le regirá en el futuro.

Como ejemplo se puede citar que ha sido demostrado que los bebes al nacer, prefieren los olores ya sabores de la comida de la que se alimentaba la madre en el embarazo. Desde el embarazo el sonido que constantemente escuchan los bebes es el de la madre, y todo lo que esta le puede trasmitir, por lo que no se recomienda reducir el nivel de estrés en el embarazo, porque este produce situaciones de tensión en el futuro bebe al nacer.

Siendo relevante lo que manifiesta esta autora relacionado al comienzo del aprendizaje en el ser humano, desde el vientre de su madre a medida que se va desarrollando durante los 9 meses, aprende significativamente y guarda en su cerebro información importante y relevante que luego la va a comparar y utilizar cuando nace al comunicarse con el mundo exterior. 


\section{Aprendiendo desde el nacimiento.}

A continuación se analiza que pasa con el niño después de que nace en su proceso de aprendizaje:

Desde el nacimiento hasta los primeros 3 años de vida, el cerebro del niño está muy activo en su proceso de aprendizaje, atento a los estímulos e interacciones significativas de: Lenguaje, tacto, contacto visual, exploración y juego, cuanto más información obtenga de estas, más fácilmente aprenderá a descifrar patrones de lenguaje, que le permitirán hacerse entender con el mundo que le rodea; desarrollando así destrezas cognitivas, emocionales y sociales que le preparan para sus primeros años de educación formal y su vida futura relacionándose con los demás (Fundacion, Bernard Van Leer, 2013).

En este apartado se manifiesta que es muy importante tener en cuenta lo que el niño aprende durante sus primeros tres años de vida, porque en este tiempo se debe formar la base de su aprendizaje en varios aspectos, para que cundo empiece sus educación académica regular pueda desarrollar sus demás capacidades con éxito.

\section{¿Cómo aprendemos?}

Cada estimulación, experiencia o conducta, permite un nuevo aprendizaje que hace que este se modifique con un nuevo conocimiento, el autor (Jensen, 2008) plantea, que cuando algún tipo de estímulo llega al cerebro, este desencadena un gran proceso, que puede ser interno o externo, luego este estimulo se distribuye a varios niveles, para luego dar lugar a la formación de una memoria potencial. Aplicado a la práctica educativa el estímulo en el aprendizaje siempre debe de tener mucha relevancia, puesto que el cerebro siempre está haciendo algo que ya sabe, o que va a conocer recién, haciendo que las vías neuronales se vuelvan cada vez más eficaces.

Para entender esta teoría que afirma el autor, se pude agregar que el aprendizaje empieza con cualquier estimulo que llega al cerebro, el mismo que desencadena una serie de procesos el cual van a producir un nuevo conocimiento que se almacena en la 
CIENCIAMATRIA

Revista Interdisciplinaria de Humanidades, Educación, Ciencia y Tecnología

Año VI. Vol. VI. N¹. Edición Especial. 2019

Hecho el depósito de ley: pp201602FA4721 ISSN-L: 2542-3029; ISSN: 2610-802X

Universidad Nacional Experimental Francisco de Miranda (UNEFM). Santa Ana de Coro. Venezuela

Dubal Edisson Salvatierra Tumbaco; Patricio Alfredo Vallejo Valdivieso; María Elena Moya Martínez

memoria, el mismo que estará disponible para ser utilizado en cualquier momento oportuno. Este tipo de estímulo puede ser diverso por ejemplo: escuchar una música, ver una película, visitar un lugar nuevo, hacer nuevas amistades, resolver un problema, etc.

\section{Relación entre aprendizaje y memoria.}

El aprendizaje es un proceso el cual modifica el conocimiento existente y por consiguiente sus capacidades de conducta y razonamiento:

Cuando hablamos de Aprendizaje y Memoria, nos referimos a dos procesos que guardan estrecha relación, y hace referencia a dos momentos de una serie de procesos mediante los cuales el cerebro maneja y elabora información proporcionada por los sentidos. Este proceso modifica el estado del conocimiento de la persona y por consecuencia sus capacidades conductuales, dicho de otra forma: como se incorpora nueva información, se modifica el estado de la memoria (Aguilar, 2001).

De esta forma el autor manifiesta que el aprendizaje se inicia cuando los sentidos captan la información, estos van a la memoria para modificar el estado de esta, la misma que le permite actuar con este nuevo conocimiento de acuerdo a lo que se requiera en el momento oportuno.

\section{El aprendizaje y la creatividad.}

Hay muchos autores que afirman que el aprendizaje debe tener una constante creatividad, para poner en práctica las inteligencias múltiples. Elisondo, (2015) plantea, que de este tema se ha investigado y que la creatividad es atributo de unos pocos elegidos, tomando como ejemplo el campo de las artes; Sin embargo investigaciones han demostrado que la creatividad es una característica de la cognición normal y que es aplicable en muchas áreas del conocimiento, por los tanto todos podemos ser creativos en cualquiera de las acciones que desarrollamos a diario, en tanto que se aplique a lo 
siguiente: que la creatividad se relaciona con la capacidad de generar nuevas ideas, apropiadas y de alta calidad.

Del análisis del autor se puede decir que todos somos capaces de ser creativos, y que este es un factor muy importante en el aprendizaje, generando nuevas ideas apropiadas a la medida que se va desarrollando el aprendizaje formal en la tabla 1, se puede observar un análisis de algunos autores del tema tratado.

\section{Habilidades cognitivas.}

Sobre las habilidades cognitivas (Gómez, 2004) se refiere a: las aptitudes que como sistema de proceso complejo del pensamiento abarca desde la captación de estímulo, el almacenaje en la memoria y su posterior utilización, por lo tanto se refiere a; las operaciones que se usan para obtener, acumular y su posterior recuperación de conocimientos, y ser usados donde y cuando se las necesite. Entre estas operaciones tenemos: (Atención = Exploración), (Comprensión = Captación de ideas), (Elaboración = analogías), (Memorización/Recuperación = Codificación / Generación de respuestas).

\section{Herencia genética y medio ambiente.}

El ser humano se va desarrollando por dos factores, el genético y el medio ambiente. La interacción entre lo biológico y la experiencia hacen que el cerebro se pueda modular desde temprana edad. Por un lado está la influencia de los genes heredados de los padres, (Rasgos físicos, temperamento, interacción, comportamiento). Por otro lado el ambiente en que se desenvuelve con las diferentes experiencias y estímulos vividos. Este proceso de neurodesarrollo es fundamental porque influye en la construcción inicial de una arquitectura cerebral, que influirá en: el comportamiento, el aprendizaje y habilidades. Estudios realizados han demostrado que ninguno influye más que el otro, ambos son fundamentales y merecen especial atención para brindar acciones concretas que permitan favorecer oportunidades de desarrollo al ser humano que está en constante aprendizaje (Campos, 2014). 
CIENCIAMATRIA

Revista Interdisciplinaria de Humanidades, Educación, Ciencia y Tecnología

Año VI. Vol. VI. N¹. Edición Especial. 2019

Hecho el depósito de ley: pp201602FA4721

ISSN-L: 2542-3029; ISSN: 2610-802X

Universidad Nacional Experimental Francisco de Miranda (UNEFM). Santa Ana de Coro. Venezuela

Dubal Edisson Salvatierra Tumbaco; Patricio Alfredo Vallejo Valdivieso; María Elena Moya Martínez

\section{Intervención Neuropsicológica.}

Es un proceso que se aplica en el ámbito escolar para diagnosticar y aplicar programas de intervención neuropsicológica, (Lobo, 2013)manifiesta: estos programas requieren de una serie de orientaciones básicas, que se aplican en la rehabilitación cognitiva para garantizar la efectividad de los mismos. También es importante tener en cuenta el desarrollo cognitivo y su correspondencia con la maduración cerebral. Todas las funciones cerebrales son muy importantes en el proceso de aprendizaje, por lo que la intervención neuropsicológica debe usar modelos teóricos, instrumentos y programas que le permitan evaluar desde los procesos más básicos hasta los más complejos y las alteraciones de aprendizajes.

\section{Cuadro 1}

Análisis de algunos autores

\begin{tabular}{|c|c|c|c|}
\hline Autor & Tema & Año & Resultado \\
\hline $\begin{array}{l}\text { Arias, } \\
\text { Liliana }\end{array}$ & $\begin{array}{l}\text { Modulo: } \\
\text { Cerebro } \\
\text { Aprendizaje. }\end{array}$ & 2011 & $\begin{array}{l}\text { El órgano de nuestro cuerpo que realiza } \\
\text { todo el aprendizaje durante toda la vida se } \\
\text { llama cerebro, que lo realiza desde antes de } \\
\text { nacer y después de nacer por el tiempo que } \\
\text { dure la vida, a través de unas células } \\
\text { llamadas neuronas. }\end{array}$ \\
\hline $\begin{array}{l}\text { Jensen, } \\
\text { Erick }\end{array}$ & $\begin{array}{l}\text { Cerebro y } \\
\text { Aprendizaje. } \\
\text { Competencias } \\
\text { e implicaciones } \\
\text { educativas. }\end{array}$ & 2008 & $\begin{array}{l}\text { Siendo el cerebro el órgano más importante } \\
\text { de nuestro cuerpo necesita energía para su } \\
\text { funcionamiento y aprendizaje, por lo tanto } \\
\text { hay que proporcionarle nutrientes } \\
\text { adecuados para que pueda funcionar bien y } \\
\text { realizar todos los procesos neuronales para } \\
\text { el cual fue creado. }\end{array}$ \\
\hline $\begin{array}{l}\text { Lagos, } \\
\text { Alejandra }\end{array}$ & $\begin{array}{l}\text { El aprendizaje } \\
\text { comienza } \\
\text { desde la } \\
\text { gestación }\end{array}$ & 2014 & $\begin{array}{l}\text { Se manifiesta que el aprendizaje empieza } \\
\text { desde el momento mismo de la concepción, } \\
\text { y que aquí se forma un patrón con } \\
\text { información que trae el niño al nacer, la cual } \\
\text { la va a relacionar con sus primeras } \\
\text { experiencias de vida. }\end{array}$ \\
\hline
\end{tabular}




\begin{tabular}{|c|c|c|c|}
\hline undación, & El aprendizaje & 2013 & $\begin{array}{l}\text { Como el niño viene en su cerebro con } \\
\text { información caraada que recibió en la }\end{array}$ \\
\hline $\begin{array}{l}\text { Beenara } \\
\text { Van Leer }\end{array}$ & $\begin{array}{l}\text { se inicia } \\
\text { temprano }\end{array}$ & & $\begin{array}{l}\text { intormación cargada que recibio en la } \\
\text { gestación, lo primero que hace es relacionar } \\
\text { esa información con el mundo exterior, y } \\
\text { luego con las diferentes interacciones que } \\
\text { realiza, va aumentando su aprendizaje y } \\
\text { obteniendo información significativa. }\end{array}$ \\
\hline guilar, L & $\begin{array}{l}\text { Aprendizaje } \\
\text { Memoria. }\end{array}$ & 2001 & $\begin{array}{l}\text { Bueno, como se ha dicho que el aprendizaje } \\
\text { se inicia en la gestación, el niño ya tiene en } \\
\text { su memoria información significativa, puesto } \\
\text { que su nuevo aprendizaje no inicia de la } \\
\text { nada, siempre habar un punto de partida } \\
\text { para un nuevo aprendizaje, que poco a poco } \\
\text { se va almacenando en su memoria, para } \\
\text { usarlo en un nuevo conocimiento. }\end{array}$ \\
\hline $\begin{array}{l}\text { isondo, } \\
\text { omina }\end{array}$ & $\begin{array}{l}\text { La creatividad } \\
\text { como } \\
\text { perspectiva } \\
\text { educativa }\end{array}$ & 2015 & $\begin{array}{l}\text { En este caso el autor manifiesta que todos } \\
\text { podemos ser creativos y esta capacidad } \\
\text { viene dada por la forma en que } \\
\text { desarrollemos nuevas ideas significativas y } \\
\text { de alta calidad. }\end{array}$ \\
\hline $\begin{array}{l}\text { Gómez, } \\
\text { José }\end{array}$ & $\begin{array}{l}\text { Neurociencia } \\
\text { Cognitiva y } \\
\text { Educacion }\end{array}$ & 2004 & $\begin{array}{l}\text { bre las habilidades cognitivas, este autor } \\
\text { anifiesta que son las operaciones que el } \\
\text { rebro realiza para captar, acumular y } \\
\text { sterior utilización de información. }\end{array}$ \\
\hline $\begin{array}{l}\text { ampos, } \\
\text { na Lucia }\end{array}$ & $\begin{array}{l}\text { Los aportes de } \\
\text { la neurociencia } \\
\text { a la atencion y } \\
\text { educacion de la } \\
\text { primera } \\
\text { infancia. }\end{array}$ & 2014 & $\begin{array}{l}\text { Sobre la genética y el medio ambiente, este } \\
\text { autor manifiesta que: La interacción entre lo } \\
\text { biológico y la experiencia diaria, permiten } \\
\text { que el cerebro se pueda moldear desde } \\
\text { temprana edad. }\end{array}$ \\
\hline $\begin{array}{l}\text { Lobo, } \\
\text { Martin } \\
\text { Pilar }\end{array}$ & $\begin{array}{l}\text { Procesos y } \\
\text { programas de } \\
\text { neuropsicologia } \\
\text { educativa. }\end{array}$ & 2013 & $\begin{array}{l}\text { La neuropsicología educativa, es un proceso } \\
\text { que se usa para diagnosticar y aplicar } \\
\text { programas de intervención neuropsicológica, } \\
\text { para rehabilitar las alteraciones de } \\
\text { aprendizaje. }\end{array}$ \\
\hline
\end{tabular}

Después de la investigación realizada se conoció que el cerebro es uno de las primeras partes del cuerpo humano que se desarrolla y aprende desde la concepción, y que un vez que el niño nace continua su aprendizaje, almacenando en su memoria la 
información significativa que le va a servir para otro aprendizaje. También hay otros factores que inciden en el aprendizaje, como la alimentación y buena nutrición que proporcionemos a nuestro cerebro mediante el torrente sanguíneo.

Hablar de cerebro y a aprendizaje es adherirse a un mundo maravilloso de las ciencias, descubrir cómo está estructurado el cerebro y cómo actúan cada una de estas partes en el proceso de aprendizaje constante.

El saber que el cerebro está conectado a cada una de las funciones motoras, visuales y auditivas y que se pueden generar funciones ejecutivas que permitan diferenciar y actuar de forma correcta a la hora que se presenta un nuevo aprendizaje en el cerebro.

Dentro del campo educativo es necesario saber de todas estas funciones que realiza el cerebro, porque de esta manera se puede comprender por qué en el aula se tiene diferentes tipos de estudiantes y que no todos aprenden de la misma manera ni al mismo ritmo.

Por otro lado la genética y el medio ambiente son dos factores importantes a la hora de moldear el cerebro a temprana edad, y que las habilidades cognitivas son ese conjunto de actividades que el cerebro realiza para: captar, almacenar y su posterior recuperación de la información.

\section{MÉTODO}

La investigación se desarrolló mediante la correlación de Pearson, lo que permitió gestionar la relación entre estructuras mentales y competencias emocionales, teniendo como fundamento lo planteado por Hernández, Fernández y Baptista (2014), de ese modo, se aplicó un instrumento de 41 ítems de cinco alternativas de repuestas en escala tipo Likert, a 21 estudiantes de la carrera de enfermería de la universidad Técnica de Manabí, sede Portoviejo - Ecuador, con la finalidad de conocer su percepción sobre las variables de investigación. 
CIENCIAMATRIA

Revista Interdisciplinaria de Humanidades, Educación, Ciencia y Tecnología

Año VI. Vol. VI. N¹. Edición Especial. 2019

Hecho el depósito de ley: pp201602FA4721 ISSN-L: 2542-3029; ISSN: 2610-802X

Universidad Nacional Experimental Francisco de Miranda (UNEFM). Santa Ana de Coro. Venezuela

Dubal Edisson Salvatierra Tumbaco; Patricio Alfredo Vallejo Valdivieso; María Elena Moya Martínez

\section{RESULTADOS}

\section{Cuadro 2}

Correlación entre estructuras mentales y competencias emocionales

\begin{tabular}{llr|r} 
& & \multicolumn{1}{c}{ EM } & \multicolumn{1}{c}{ CE } \\
\hline \multirow{2}{*}{ EM } & Correlación de Pearson & 1 &, 183 \\
\cline { 2 - 4 } & Sig. (bilateral) & & 426 \\
\cline { 2 - 4 } & $\mathrm{N}$ & 21 & 21 \\
\hline \multirow{2}{*}{ CE } & Correlación de Pearson &, 183 & 1 \\
\cline { 2 - 4 } & Sig. (bilateral) &, 426 & \\
\hline N & 21 & 21 \\
\hline
\end{tabular}

Los resultados entre estructuras mentales (EM) y competencias emocionales (CE), demuestran una relación positiva baja de ,183 lo cual conlleva a proceder la realización de la prueba de hipótesis:

Se acepta a:

H1: Existe relación entre ambas variables

Y se rechaza a:

H0: No existe relación entre ambas variables

\section{DISCUSIÓN}

A partir de la correlación obtenida, se evidencia que al promoverse las estructuras mentales en función de la generación de competencias emocionales, se puede incentivar el aprendizaje significativo en los estudiantes, al respecto Vallejo Valdivieso, Zambrano Pincay, Vallejo Pilligua \& Bravo Cedeño (2019), indican que es necesario 
que el docente estimule el cerebro desde una perspectiva emocional positiva con la finalidad de sensibilizar, en la generación de nuevos conocimientos, así mismo, Vallejo Valdivieso, Zambrano Pincay, Vallejo Pilligua \& Bravo Cedeño (2019), complementan al señalar que la persona realiza conexiones con la realidad social en función de fusionar teoría y praxis con la comprensión de nuevos estadios de conocimiento en función de articular el aprendizaje significativo.

\section{CONCLUSIONES}

El aprendizaje significativo en contextos de estudios universitarios de enfermería puede ser incentivado desde una adecuada estimulación de las estructuras mentales en relación a las competencias emocionales, así se generan una serie de actividades que permiten al estudiante conciliar no solo teoría y significación social a los fenómenos abordados en el aprendizaje, sino, también el fomento de una adecuada personalidad promovida desde lo emocional como ente que permite configurar la integralidad de la persona en consonancia con ser reflexivo, critico, en situación de combinarse en su rol ciudadano desde fu rol estudiantil, situación que permite progresivamente comprometerse en la transformación social de su entorno.

\section{REFERENCIAS CONSULTADAS}

1. Aguilar, L. (2001). Aprendizaje y Memoria. Madrid: Editores: M.Gadea, L. Perez.

2. Bisquerra, R. (2007). Un modelo de competencias emocionales. Recuperado de http://www.rafaelbisquerra.com/es/competencias-emocionales/competenciasocial/81-competencias-emocionales/102-un-modelo-competenciasemocionales.html

3. Briceño Ruperti, M., Vallejo Valdivieso, P., \& Moya Martínez, M. (2019). Estructuras mentales y competencia mediática en el aprendizaje significativo. CIENCIAMATRIA, 5(9), 680-695. https://doi.org/10.35381/cm.v5i9.258 
CIENCIAMATRIA

Revista Interdisciplinaria de Humanidades, Educación, Ciencia y Tecnología

Año VI. Vol. VI. N¹. Edición Especial. 2019

Hecho el depósito de ley: pp201602FA4721

ISSN-L: 2542-3029; ISSN: 2610-802X

Universidad Nacional Experimental Francisco de Miranda (UNEFM). Santa Ana de Coro. Venezuela

Dubal Edisson Salvatierra Tumbaco; Patricio Alfredo Vallejo Valdivieso; María Elena Moya Martínez

4. Campos, A. L. (2014). Los aportes de la neurociencia a la atencion y educacion de la primera infancia. Bolivia: Cerebrum Ediciones.

5. Elisondo, R. (2015). La creatividad como perspectiva educativa. Actualidades Educativas en Educacion, 23.

6. Fundacion, Bernard Van Leer. (2013). El aprendizaje se inicia temprano. La haya, Paises bajos.: Teresa Moreno.

7. Gómez, J. (2004). Neurociencia Cognitiva y Educacion. Lambayeque: Fondo Editorial FACHSE.

8. Hernández, R. Fernández, C., y Baptista, P. (2014). Metodología de la investigación. México, Mc Graw Hill Hispanoamericana. Hill Internacional.

9. Jensen, E. (2008). Cerebro y Aprendizaje. Competencias e implicaciones educativas. Madrid: Narcea, S.A. Ediciones.

10.Lagos, A. (2014). Univision.com / Educacion. Obtenido de Univision.com / Educacion: $\quad$ https://www.univision.com/noticias/educacion/el-aprendizajecomienza-desde-la-gestacion

11. Lobo, M. P. (2013). Procesos y programas de neuropsicologia educativa. Madrid: Secretaria General Tecnica - Ministerio de Educacion Cultura y Deportes

12. Morgado, C., Aldana Zavala, J., \& Isea Argüelles, J. (2019). Gerencia transformacional desde el accionar docente directivo para el abordaje del Contexto Escolar. CIENCIAMATRIA, 5(9), 48-72. https://doi.org/10.35381/cm.v5i9.99

13. Vallejo Valdivieso, P., Zambrano Pincay, G., Vallejo Pilligua, P., \& Bravo Cedeño, G. (2019). Estructuras mentales en la construcción de aprendizaje significativo. CIENCIAMATRIA, 5(8), 228-241. Recuperado a partir de http://cienciamatriarevista.org.ve/index.php/cm/article/view/257

14. Vallejo Valdivieso, P., Zambrano Pincay, G., Vallejo Pilligua, P., \& Bravo Cedeño, G. (2019). Importancia del Conectivismo en la inclusión para mejorar la Calidad Educativa ante la tecnología moderna. Revista Arbitrada Interdisciplinaria Koinonía, 4(8), 523-543. doi:http://dx.doi.org/10.35381/r.k.v4i8.297 


\section{REFERENCES CONSULTED}

1. Aguilar, L. (2001). Learning and memory. Madrid: Publishers: M. Gadea, L. Perez.

2. Bisquerra, R. (2007). A model of emotional competencies. Recovered from http://www.rafaelbisquerra.com/es/competencias-emocionales/competenciasocial/81-competencias-emocionales/102-un-modelo-competenciasemocionales.html

3. Briceño Ruperti, M., Vallejo Valdivieso, P., \& Moya Martínez, M. (2019). Mental structures and media competence in meaningful learning. SCIENCE, 5 (9), 680695. https://doi.org/10.35381/cm.v5i9.258

4. Campos, A. L. (2014). The contributions of neuroscience to the attention and education of early childhood. Bolivia: Cerebrum Editions.

5. Elisondo, R. (2015). Creativity as an educational perspective. Educational News in Education, 23.

6. Foundation, Bernard Van Leer. (2013). Learning starts early. The Hague, Netherlands .: Teresa Moreno.

7. Gómez, J. (2004). Cognitive Neuroscience and Education. Lambayeque: FACHSE Editorial Fund.

8. Hernández, R. Fernández, C., and Baptista, P. (2014). Investigation methodology. Mexico, Mc Graw Hill Hispanic American. Hill International

9. Jensen, E. (2008). Brain and learning. Educational competencies and implications. Madrid: Narcea, S.A. Editions

10.Lagos, A. (2014). Univision.com / Education. Obtained from Univision.com / Education: https://www.univision.com/noticias/educacion/el-aprendizajecomienza-desde-la-gestacion

11. Lobo, M. P. (2013). Educational neuropsychology processes and programs. Madrid: General Technical Secretary - Ministry of Education Culture and Sports

12. Morgado, C., Aldana Zavala, J., \& Isea Argüelles, J. (2019). Transformational 


\section{CIENCIAMATRIA}

Revista Interdisciplinaria de Humanidades, Educación, Ciencia y Tecnología

Año VI. Vol. VI. N¹. Edición Especial. 2019

Hecho el depósito de ley: pp201602FA4721

ISSN-L: 2542-3029; ISSN: 2610-802X

Universidad Nacional Experimental Francisco de Miranda (UNEFM). Santa Ana de Coro. Venezuela

Dubal Edisson Salvatierra Tumbaco; Patricio Alfredo Vallejo Valdivieso; María Elena Moya Martínez

management from the directive teaching actions to address the School Context. SCIENCE, 5 (9), 48-72. https://doi.org/10.35381/cm.v5i9.99

13. Vallejo Valdivieso, P., Zambrano Pincay, G., Vallejo Pilligua, P., \& Bravo Cedeño, G. (2019). Mental structures in the construction of meaningful learning. SCIENCE, $\quad 5 \quad(8), \quad 228-241 . \quad$ Recovered from http://cienciamatriarevista.org.ve/index.php/cm/article/view/257

14. Vallejo Valdivieso, P., Zambrano Pincay, G., Vallejo Pilligua, P., \& Bravo Cedeño, G. (2019). Importance of Connectivism in inclusion to improve Educational Quality in the face of modern technology. Interdisciplinary Arbitrated Review Koinonía, 4 (8), 523-543. doi: http: //dx.doi.org/10.35381/r.k.v4i8.297

(C2019 por los autores. Este artículo es de acceso abierto y distribuido según los términos y condiciones de la licencia Creative Commons Atribución-NoComercial-Compartirlgual 4.0 Internacional (CC BY-NC-

SA 4.0) (https://creativecommons.org/licenses/by-nc-sa/4.0/). 